\title{
Additive main effects and multiplicative interaction analysis and clustering of environments and genotypes in malting barley
}

\author{
Muluken Bantayehu*, Jemal Esmael and Yihenewu Awoke \\ Adet Agricultural Research Center, P. O. Box 08, Bahirdar, Ethiopia.
}

Accepted 18 April, 2011

\begin{abstract}
Grain yield of twenty malting barley genotypes planted in four locations over three years were used to study the effect of genotypes, environments and genotype by environment interaction. Additive main effects and multiplicative interaction (AMMI) analysis was made for yield estimation, to understand the genotype by environment (GxE) interaction patterns, clustering of environments and genotypes into homogenous subunits and to study genotypic yield stability. AMMI showed that genotypes 1, 3 and 9 among high, medium and low yielder groups, respectively provided the most stable genotypes when viewed along with the first two interaction components. The environments showed high variability both in mean yield and interaction patterns, and Da-07 and La-05 were found to be less interactive with all genotypes. Clustering of AMMI estimate values grouped genotypes into five clusters and environments into four clusters. Genotypes numbers 2, 7, 17, HB-52 and HB-12, are unique as they are grouped differently from all the rest genotypes. Ethiopia is classified into 18 major agro ecological zones and 49 subagroecological zones and it is essential to cluster similar environments and develop varieties for each target environments. Consequently the genotypes EH1609-F5.B3-10 and EH1603-F5.B1-4 were stable and high yielders across the tested agroecologies of north western Ethiopia.
\end{abstract}

Key words: Malting barley, genotype by environment $(G \times E)$ interaction, additive main effects and multiplicative interaction (AMMI), stability, clustering.

\section{INTRODUCTION}

Production of malting barley started recently and is expected to increase rapidly in north western Ethiopia. Malting barley is mostly grown as an industrial crop. It is a source of alcohol, protein and enzymes in the preparation of beer. Adet agricultural research center has conducted variety traits since 1985 to identify potential production environments and develop stable high yielding varieties. However, the genotype by environment (GxE) interaction structure is an important aspect of both plant breeding programs and the introduction of new crop commodities. The GxE interaction may arise when specified genotypes are grown in diverse environments
(Zobel, 1990). A significant GxE interaction for quantitative traits such as grain yield can seriously limit efforts in selecting superior genotypes for both new crop introduction and improved cultivar development (Kang, 1990). Statistically, a significant interaction encountered in analysis of two-way classification (for example, cultivar $x$ location) would reduce the usefulness of subsequent analysis of means and inferences that would otherwise be valid.

The additive main effect and multiplicative interaction, widely known as the AMMI model, combines regression analysis of variance (ANOVA) for additive main effects 
with principal components analysis (PCA) for multiplicative structure within the interaction (Gauch and Zobel, 1996). According to Zobel et al. (1988), analysis of variance fails to detect a significant interaction component, PCA fails to identify and separate the significant genotype and environment main effects, and linear regression models accounts for only a small portion of the interaction sum of squares. But, additive main effects and multiplicative interaction methods, which combines analysis of variance of genotype and environmental main effects with principal components analysis of the GxE interaction brought in a unified approach (Gauch, 1988; Zobel et al., 1988; Gauch and Zobel, 1996). AMMI analysis reveals a highly significant interaction component that has clear agronomic meaning and it has no specific design requirement, except for a two way data structure. In AMMI, the additive main effect portion is separated from interaction by ANOVA model. Then the principal component analysis (PCA) that provides a multiplicative model (Gabriel, 1971; Zobel et al., 1988) is applied to analyze the interaction effect from the additive ANOVA model.

The PCA of AMMI partitions GxE interactions into several orthogonal axes, the interaction principal component axes (IPCA). There are several possible AMMI models characterized by a number of significant PCA axes ranging from zero (AMMI-0, that is, additive model) to a minimum between ( $g-1)$ and $(I-1)$ where $g=$ number of genotypes and $\mathrm{I}=$ number of locations. The full model (AMMI-F) with the highest number of PCA axes provides a perfect comparison between expected and observed data. Guach and Zobel (1996) showed that AMMI 1 with IPCA 1 and AMMI 2 with IPCA 1 and IPCA 2 are usually selected and the graphical representation of axes, either as IPCA 1 or IPCA 2 against main effects or IPCA 1 against IPCA 2 which is usually the most appropriate. The result can be graphically represented in an easily interpretable and informative biplot, which shows both main effects and GxE interactions. Thus, the objectives of this study include; To use AMMI analysis for yield estimation, understand the GxE interaction patterns, clustering of environments and genotypes into homogenous subunits, and to Identify stable and high yielding genotype

\section{MATERIALS AND METHODS}

\section{Field trail}

The field experiment was carried out with 20 advanced malting barley genotypes along with standard checks where $01=\mathrm{EH} 1609$ F5.B3-10 (G1); $02=37622$ (G2); $03=118173$ (G3); 04 = Bekoji sel-8 (G4); $05=118007$ (G5); $06=\mathrm{EH} 1510-\mathrm{F} 6.10 \mathrm{H} .3$ (G6); $07=$ 108932 (G7); $08=118173$ (G8); $09=118146$ (G9); $10=\mathrm{EH} 1746$ F6.B2-109 (G10); $11=$ EH1606-F5.B2-7 (G11); $12=\mathrm{EH} 1601-$ F5.B2-2 (G12); $13=$ EH1603-F5.B1-4 (G13); $14=$ EH1612F5.B3.13 (G14); $15=$ HB1533- sels (G15), and $16=$ Miscal-2 (G16); 17 = Miscal-1 (G17); 18 = HB-242-sels (G18), along with 19 $=\mathrm{HB}-52(\mathrm{G} 19) ; 20=\mathrm{HB}-120$ (G20). The experiments were conducted in four representative sites of barley growing agroecologies during three main rain growing seasons (2004, 2005 and 2007); $A=$ Adet, 2004; $B=$ Adet, 2005; $C=$ Adet, 2007; $D=$ Debretabor, 2004; E = Debretabor, 2005; F = Debretabor, 2007; G = Dabat, 2004; H = Dabat, 2005; I = Dabat, 2007; J = Laygaint, 2004; K = Laygaint, 2005; L = Laygaint, 2007. At each site, the genotypes were planted in a randomized block design with three replications. Sowing was done by hand in plots of $3 \mathrm{~m}^{2}$ with six rows of $2.5 \mathrm{~m}$ length and $20 \mathrm{~cm}$ row spacing. The four middle rows of $2 \mathrm{~m}^{2}$ harvested. Fertilizer application was $41 \mathrm{~N} \mathrm{kgha}^{-1}$ and 46 $\mathrm{P}_{2} \mathrm{O}_{5} \mathrm{~kg} \backslash \mathrm{ha}$ at planting. Grain yield $\left(\mathrm{kgha}^{-1}\right)$ was calculated by converting the grain yields obtained from plots to hectares.

\section{Statistical analysis}

Analysis of variance (ANOVA) was used to determine differences among the genotypes $(G)$, environments (E) and genotype by environment interaction (GxE). Additive main effects and multiplicative interaction (AMMI) model analysis was performed. The AMMI model is

$Y_{i j}=\mu+g_{i}+e_{j}+\sum_{k=1}^{n} \lambda_{k} \alpha_{i k} \gamma_{j k}+R_{I J}$

Where, ${ }{ }_{i j}$ is the yield of the ith genotype in the th environment; $g_{i}$ is the mean of the ith genotype minus the grand mean; ${ }^{e_{j}}$ is the mean of the jth environment minus the grand mean; $\lambda_{k}$ is the square root of the eigenvalue of the PCA axis K; $\alpha_{i k}$ and $\gamma_{j k}$ are the principal component scores for PCA axis $k$ of the ith genotype and the jth environment, respectively, and $R_{i j}$ is the residual. Environmental and genotype PCA scores are expressed as a unit vector times the square root of $\lambda_{k}$ (that is, environment PCA score $=\lambda_{k}^{0.5} \alpha_{i k}$; genotype PCA score $=\lambda_{k}^{0.5} \alpha_{i k}$ ) (Zobel et al., 1988). The degree of freedom (df) for the IPCA axes was calculated based on Gollob (1968) method. Df $=\mathrm{G}+\mathrm{E}-1-2 \mathrm{n}$; where, Ggenotypes, E- environments and $n$ - number of IPCA axis.

To show a clear insight into specific genotype $x$ environment interaction combinations and the general pattern of adaptation, a biplot of genotypes and environments (Kempton, 1984) was done for some important traits. In the biplots, the first IPCA was used as the ordinate (Y-axis) and the second IPCA represented abscissa (X-axis) in AMMI 2. Cluster analysis of standardized AMMI estimated that, grain yield was carried out. For each environment, standardization to a mean of zero and a unit standard deviation was performed to adjust for yield differences between environments. This causes environmental clustering to be determined by the relative performance of genotypes within environments. The ward or incremental sum of squares method was used as a clustering method to group genotypes that have similar environment classes, and vice versa.

\section{RESULTS}

\section{AMMI analysis of variance}

The analysis of variance showed significant effects for genotype (G), environment (E), and GxE interaction 
Table 1. Additive main effects and multiplicative interactions (AMMI) analysis of variance for grain yield of 20 genotype of malting barley across 12 environments.

\begin{tabular}{lccc}
\hline Source & d.f. & Mean squares & Variance explained (\%) \\
\hline Total & 719 & - & - \\
Treatment & 230 & 3868773.39 & 92.39 \\
Blocks & 24 & 60865505.0 & 3.91 \\
Environment & 11 & $60865505.0^{* *}$ & 75.24 \\
Genotypes & 19 & $4365559.1^{* *}$ & 9.32 \\
Interactions & 209 & $657185.2^{* *}$ & 15.44 \\
IPCA 1 & 29 & $1695438.4^{* *}$ & 35.80 \\
IPCA 2 & 27 & $1123189.2^{* *}$ & 22.08 \\
IPCA 3 & 25 & $637724.8^{* *}$ & 11.61 \\
IPCA 4 & 23 & $554398.6^{* *}$ & 9.28 \\
IPCA 5 & 21 & $439107.1^{* *}$ & 6.71 \\
Error & 456 & 78097.8 & 3.70 \\
\hline
\end{tabular}

${ }^{* *}$ Highly significant at the 0.01 probability level, ${ }^{*}$ significant at the 0.05 probability level, d.f., degree of freedom; IPCA, principal component axis for interaction.

(Table 1). The result showed that, $69 \%$ of the total sum of squares (SS) was attributed to environmental effects; only $8.6 \%$ genotype and $14.3 \%$ were attributed to genotypes and GxE interaction effects, respectively. Results from analysis of multiplicative effects also showed that the first interaction principal component axis (IPCA 1) captured $35.80 \%$ of the interaction SS in $13.9 \%$ of the interaction degree of freedom (df). Similarly, the IPCA 2, IPCA 3, and IPCA 4 explained a further 22.08, 11.61 and $9.28 \%$ of the GxE interaction SS, respectively. An F-test at $P=0.01$ revealed that the first five principal component axes of the interaction were significant for the model. However, the prediction assessment indicated that AMMI 2 with only two interaction principal component axes was the best predictive model (Zobel et al., 1988). Further interaction principal component axes captured mostly noise and therefore did not help to predict validation observations. In total, the AMMI 2 model $(\mathrm{G}+\mathrm{E}+\mathrm{IPCA} 1$ and IPCA 2) contained $94 \%$ of the total SS, indicating that, the AMMI model fits the data well, and validates the use of AMMI 2. Thus, the interaction of the twenty genotypes with twelve environments was best predicted by the first two principal components of genotypes and environments with similar signs of their IPCA scores which interact positively for the trait.

Scores of genotypes and environments to the first IPCA axis are presented in Table 2. The IPCA scores of a genotype provide indicators of the stability of a genotype across environments (Purchase, 1997). Regardless of the positive or negative signs, genotypes with large scores have high interactions (unstable), whereas genotypes with small IPCA scores close to zero have small interactions and are stable (Zobel et al., 1988). The lowest IPCA 1 was observed for genotype $\mathrm{G} 1$ followed by G3 and G19, and IPCA 2 was lowest for genotypes G3, G11, and G2 (Table 2). According to IPCA 1, G1 was the most stable genotype with the mean yield (2795.53 $\mathrm{kg} \backslash \mathrm{ha})$ higher than the grand mean (2178.65 $\mathrm{kg} \backslash \mathrm{ha})$. The highest IPCA 1 was given by G17 followed by $\mathrm{G} 2$ and G12 and the highest IPCA 2 was scored by G5 followed by $\mathrm{G} 18$ and $\mathrm{G} 7$, which had mean yields nearly equal to the grand mean.

\section{AMMI biplots}

Environments with higher IPCA scores discriminate among genotypes more than environments with lesser scores (Zobel et al., 1988; Kempton, 1984). Ad-04, Ad05, Ad-07, Da-07, La-05 and La-07 environments discriminate less among genotypes as their IPCA 1 score were less whereas Db-04, La-05 and Da-04 discriminate more among genotypes (Figure 1).

The IPCA scores of environments in AMMI analysis were considered to assess the behavior of each environment in GxE interaction. Ideal test environments should have large IPCA 1 scores (more discriminating of genotypes) and near zero IPCA 2 scores (more representatives of average environments) (Yan, 1999; Yan et al., 2000; Yan and Rajcan, 2002). According to environmental IPCA 1 scores, environments Ad-04, Da07 and La-07 were more stable and had lower GxE interaction but $\mathrm{La}-07$ and $\mathrm{Da}-07$ had low yield performance, whereas the highest IPCA 1 scores belonged to Db-04, La-04 and Da-07. According to IPCA 1 , environments $\mathrm{Db}-04$ were ideal environments for selecting genotypes with specific adaptation to high input conditions. By using this method, environment Da-07 followed by La-07 and Db-07 had the highest stability with the least combination of GxE interaction, whereas environment $\mathrm{Db}-04$ with the highest GxE value had the highest genotypic response. 
Table 2. Mean grain yield $\left(\mathrm{kgha}^{-1}\right)$ and first and second principal component analysis (PCA) scores of twenty genotypes grown in twelve environments.

\begin{tabular}{|c|c|c|c|c|c|c|c|c|c|c|c|c|c|c|c|}
\hline \multirow{2}{*}{ Genotype } & \multicolumn{12}{|c|}{ Environment } & \multirow{2}{*}{ Mean } & \multirow{2}{*}{ PCA 1} & \multirow{2}{*}{ PCA 2} \\
\hline & Ad-04 & Ad-05 & Ad-07 & Db-04 & Db-05 & Db-07 & Lg-04 & Lg-05 & Lg-07 & Da-04 & Da-05 & Da-07 & & & \\
\hline $\mathrm{G} 1$ & 4225.0 & 3548.3 & 3763.7 & 4164.0 & 3552.0 & 2889.7 & 1774.0 & 1805.7 & 1949.0 & 2869.7 & 885.5 & 2120.3 & 2795.5 & 0.3 & 6.9 \\
\hline G2 & 2592.0 & 1930.0 & 2138.0 & 1356.3 & 1881.3 & 2511.0 & 904.8 & 767.7 & 1223.5 & 1743.7 & 772.7 & 1309.3 & 1594.2 & 26.6 & 2.5 \\
\hline G3 & 3520.0 & 3296.0 & 3285.0 & 3370.0 & 3878.7 & 2830.3 & 1364.7 & 712.1 & 1413.0 & 1997.7 & 1663.7 & 1318.3 & 2387.3 & -1.9 & -0.6 \\
\hline G4 & 2460.0 & 2465.7 & 2340.0 & 3345.3 & 2395.3 & 2598.7 & 1377.0 & 997.3 & 1295.7 & 1964.7 & 1029.3 & 806.7 & 1923.0 & 10.1 & -6.5 \\
\hline G5 & 2785.7 & 2415.0 & 2477.0 & 3964.7 & 4614.7 & 2905.0 & 1562.3 & 735.8 & 805.2 & 1905.7 & 841.2 & 1857.7 & 2239.1 & -6.9 & -27.9 \\
\hline G6 & 3414.0 & 2829.3 & 2998.3 & 4061.0 & 3701.7 & 3942.0 & 406.3 & 834.3 & 1970.7 & 2052.3 & 175.3 & 1619.7 & 2333.7 & -17.6 & -12.6 \\
\hline G7 & 3395.7 & 2617.0 & 2883.3 & 2362.0 & 2190.3 & 2063.0 & 1235.3 & 1564.0 & 920.2 & 1488.7 & 622.3 & 522.0 & 1821.9 & 11.6 & 20.8 \\
\hline G8 & 3639.7 & 3279.0 & 3336.0 & 3436.7 & 3693.7 & 2625.3 & 863.5 & 1159.7 & 1794.7 & 2142.0 & 920.8 & 1257.0 & 2345.6 & -4.5 & 5.7 \\
\hline G9 & 3580.3 & 3213.7 & 3273.7 & 3085.7 & 3616.7 & 2801.0 & 716.2 & 771.8 & 1073.2 & 2236.0 & 1271.5 & 1218.0 & 2238.1 & -3.3 & 3.1 \\
\hline G10 & 3633.7 & 2808.7 & 3098.3 & 3851.3 & 3812.3 & 3308.3 & 1116.2 & 607.7 & 894.4 & 2360.7 & 1091.9 & 497.3 & 2256.8 & -10.7 & -7.8 \\
\hline G11 & 3732.7 & 3190.0 & 3338.3 & 3589.0 & 3887.0 & 3386.0 & 769.2 & 1165.2 & 1078.0 & 1895.0 & 987.8 & 1181.3 & 2349.9 & -13.1 & 0.7 \\
\hline G12 & 4103.7 & 3806.0 & 3832.0 & 4427.3 & 3113.3 & 3460.3 & 822.3 & 1505.3 & 1529.7 & 1685.7 & 660.8 & 1127.3 & 2506.2 & -21.4 & 15.2 \\
\hline G13 & 3933.0 & 3962.7 & 3824.7 & 3918.7 & 4038.7 & 2645.3 & 1289.7 & 1678.0 & 1611.0 & 2196.0 & 1068.8 & 1785.3 & 2662.6 & -8.6 & 11.0 \\
\hline G14 & 3527.7 & 3221.3 & 3251.0 & 3563.0 & 3629.0 & 3135.3 & 1429.0 & 2154.3 & 1340.5 & 1905.0 & 840.0 & 1117.7 & 2426.0 & -3.2 & 5.9 \\
\hline G15 & 3583.3 & 3248.7 & 3293.0 & 3820.3 & 3654.3 & 2948.0 & 1344.0 & 1241.7 & 1823.7 & 1965.3 & 600.2 & 247.7 & 2314.2 & -9.6 & 5.7 \\
\hline G16 & 2590.3 & 1925.0 & 2134.7 & 2861.7 & 3426.7 & 2348.0 & 1177.7 & 905.2 & 1388.5 & 2480.0 & 1055.6 & 687.5 & 1915.0 & 14.1 & -16.1 \\
\hline G17 & 2066.0 & 1417.0 & 1618.7 & 1545.0 & 1865.0 & 1446.7 & 1481.3 & 529.9 & 963.1 & 2799.7 & 212.5 & 539.6 & 1373.7 & 37.9 & -7.5 \\
\hline G18 & 2593.0 & 2457.0 & 2402.0 & 4377.7 & 3803.0 & 3251.7 & 1022.8 & 1386.0 & 1376.2 & 1886.7 & 607.8 & 1231.0 & 2199.6 & -10.1 & -21.8 \\
\hline G19 & 3620.7 & 2983.3 & 3179.0 & 2777.3 & 3112.0 & 1683.3 & 1420.3 & 1198.7 & 1408.7 & 1392.3 & 435.7 & 623.2 & 1986.11 & 3.0 & 19.7 \\
\hline G20 & 2608.7 & 3031.0 & 2696.7 & 1838.0 & 3657.0 & 2515.7 & 465.6 & 1350.7 & 995.1 & 1727.7 & 879.0 & 1088.9 & 1904.45 & 7.1 & 3.8 \\
\hline Mean & 3280.0 & 2882.0 & 2958.0 & 3286.0 & 3376.0 & 2765.0 & 1127.0 & 1153.0 & 1343.0 & 2035.0 & 831.0 & 1108.0 & & & \\
\hline PCA 1 & 9.0 & 12.9 & 11.0 & 34.7 & 18.6 & 13.5 & -26.7 & -11.2 & -10.8 & -25.4 & -16.3 & -9.2 & & & \\
\hline PCA 2 & 23.8 & 23.2 & 23.5 & -16.7 & -21.5 & -16.3 & -3.4 & 13.7 & 1.9 & -13.7 & -3.9 & -10.7 & & & \\
\hline
\end{tabular}

The interaction of genotypes with environments was best predicted by the first two principal components of genotypes and environments and with similar signs of their IPCA scores, interact positively for that trait. AMMI 2 biplot as shown in Figure 2 has four sections. The locations fall into four sections; genotype G12, G19 and G13 had good adaptation for locations Adet: genotype G13, G1 and G10 were good for location Debretabor; G2 and was good for Laygaint; and for Dabat genotypes G16 was good. Genotypes G1, G3, G9 and G4 located near the plot origin have low GxE interaction than the vertex genotypes and thus, stable. Genotypes G5, G17 and G7 located far from the vertex were temporally and spatially unstable.

\section{AMMI recommendation}

The mean yield of the genotypes across 12 environments ranged from $1373.73 \mathrm{~kg} / \mathrm{ha}$ to $2795.53 \mathrm{~kg}$ lha (Table 1). The difference in the ranking of genotypes across environments indicated the presence of GxE interaction, which was confirmed by the significant effect of the GxE interaction (explaining $15.44 \%$ of the $G+E+G x E$ in AMMI model). Genotype G1 was presented in the top five ranks in 11 out of 12 environments and was identified as the dominant genotype in 5 environments followed by $\mathrm{G} 13$ that appeared in 


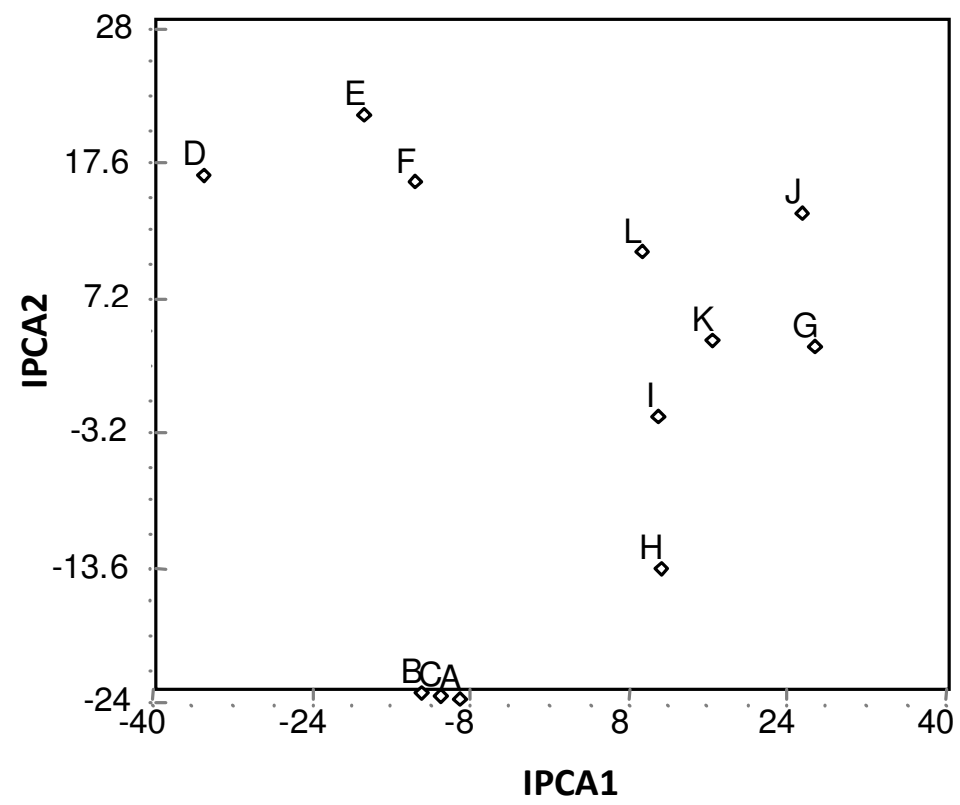

Figure 1. Biplot of IPCA 1 and IPCA 2 scores of environments included in northwestern Ethiopian variety evaluation trial in 2004, 2005 and 2007 main rain cropping season. (A, Adet, 2004; B, Adet, 2005; C, Adet, 2007; D, Debretabor, 2004; E, Debretabor, 2005; F, Debretabor, 2007; G, Dabat, 2004; H, Dabat, 2005; I, Dabat, 2007; J, Laygaint, 2004; K, Laygaint 2005; L, Laygaint, 2007).

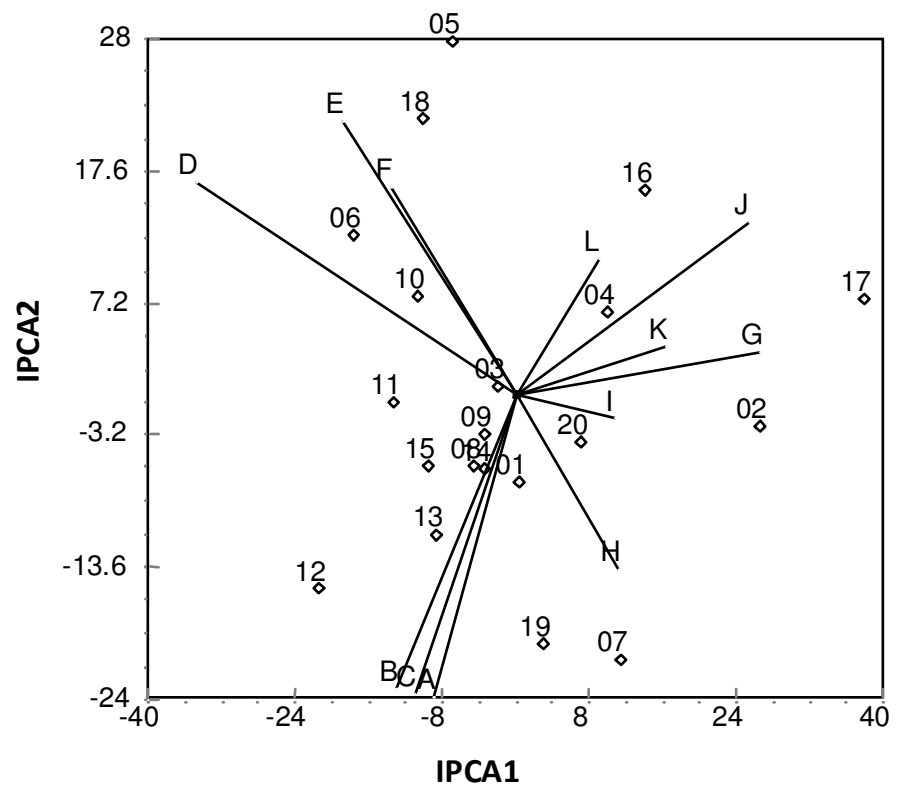

Figure 2. Biplot IPCA 1 and IPCA 2 of twenty genotypes grown at four locations in 2004, 2005 and 2007 main cropping season. $01=$ EH1609-F5.B3-10(G1); 02 = 37622 (G2); 03 = 118173(G3); 04 = Bekoji sel-8 (G4); 05 = 118007 (G5); 06 = EH1510-F6.10H.3 (G6); $07=108932(\mathrm{G} 7) ; 08=118173(\mathrm{G} 8) ; 09=118146$ (G9); $10=$ $\mathrm{EH} 1746-\mathrm{F} 6 . \mathrm{B} 2-109$ (G10); $11=\mathrm{EH} 1606-\mathrm{F} 5 . \mathrm{B} 2-7$ (G11); $12=$ EH1601-F5.B2-2 (G12); $13=$ EH1603-F5.B1-4 (G13); $14=$ EH1612-F5.B3.13 (G14); $15=$ HB1533-sels (G15), and $16=$ Miscal2 (G16); 17 = Miscal-1 (G17); $18=$ HB-242-sels (G18), $19=$ HB-52 (G19); 20 = HB-120 (G20) (A, Adet, 2004; B, Adet, 2005; C, Adet, 2007; D, Debretabor, 2004; E, Debretabor, 2005; F, Debretabor, 2007; G, Dabat, 2004; H, Dabat, 2005; I, Dabat, 2007; J, Laygaint, 2004; K, Laygaint, 2005; L, Laygaint, 2007). 
Table 3. Environment grouping using average yield, the top 5 yielding genotypes and the expected yield improvement when using the first AMMI recommended genotype.

\begin{tabular}{|c|c|c|c|c|c|c|c|}
\hline \multirow{2}{*}{ Environment } & \multirow{2}{*}{ Mean yield } & \multicolumn{5}{|c|}{ AMMI genotype recommendation } & \multirow{2}{*}{$\begin{array}{c}\text { Yield } \\
\text { improvement }\end{array}$} \\
\hline & & $1^{\text {st } A}$ & $2^{\text {nd }}$ & $3^{\text {rd }}$ & $4^{\text {th }}$ & $5^{\text {th }}$ & \\
\hline A & 3280 & G12 (4162) & G13 (4103) & G1 (4058) & G14 (3698) & G15 (3638) & 882 \\
\hline B & 2882 & G12 (3838) & G13 (3731) & G1(3654) & G14 (3308) & G15 (3274) & 956 \\
\hline C & 2958 & G12 (3877) & G13 (3794) & G1 (3733) & G14 (3380) & G15 (3333) & 919 \\
\hline D & 3286 & G6 (4262) & G12 (4102) & G5 (4049) & G18 (4019) & G11 (3902) & 976 \\
\hline$E$ & 3376 & G5 (4163) & G6 (4130) & G18 (4053) & G1 (3840) & G10 (3820) & 787 \\
\hline $\mathrm{F}$ & 2765 & G5 (3372) & G6 (3363) & G18 (3277) & G1 (3266) & G13 (3185) & 607 \\
\hline $\mathrm{G}$ & 1127 & G1 (1729) & G17 (1356) & G13 (1346) & G16 (1294) & G3 (1288) & 602 \\
\hline $\mathrm{H}$ & 1153 & G1 (1868) & G13 (1692) & G12 (1450) & G14 (1446) & G8 (1347) & 715 \\
\hline I & 1343 & G1 (1976) & G13 (1754) & G14 (1566) & G3 (1530) & G8 (1471) & 633 \\
\hline J & 2035 & G1 (2565) & G16 (2350) & G5 (2301) & G17 (2295) & G3 (2205) & 530 \\
\hline K & 831 & G1 (1426.2) & G13 (1132.8) & G3 (1011.8) & G14 (1011.8) & G8 (902.5) & 595.2 \\
\hline L & 1108 & G1 (1654) & G5 (1403) & G13 (1395) & G3 (1306) & G18 (1270) & 546 \\
\hline Average & 2178.7 & 2907.7 & & & & & 729.0 \\
\hline
\end{tabular}

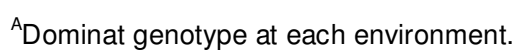

the top five ranks in 9 out of 12 environments and G12 appeared in the top five ranks in 5 of 12 environments which was the dominant genotype in 2 environments; G5 was the best in 2 environments and appeared in the top five ranks in 5 of 12 environment; G6 ranked in the top five ranks in 5 environments; G14 appeared in 6 environments within the top five ranks; $G 8$ was the top five ranks in 4 of 12 environments; and G3 appeared in the top five ranks in 3 environments. Other genotypes that were not classified as dominant, but appeared once or twice in the top five ranks across 12 environments were G10, G3, G17, and G6. Table 3 also serves to illustrate the importance of recommending the right genotype for each environment.

\section{Clustering of AMMI values}

Genotypes and environments were clustered using AMMI 2 adjusted values. Dendrogram depicting clustering of genotypes and environments are presented in Figures 3 and 4 accordingly. At the two group level of genotype clustering, five relatively poor adaptation genotypes number 2, 17, 20, 7 and 19 were discriminated and clustered from the remaining genotypes. These genotypes are characterized by low yield with high level of interaction with the environment. However, the second group of the remaining genotypes is intermediate to high yielding potential with PCA 1 and PCA 1 score of 14.1 to -27.9 .

The ward or incremental sum of squares clustering method strongly depicted a class of five sub clusters sets. Splitting down the main first branches of the dendrogram resulted in two sub clusters (Figure 4). The first sub cluster of the first group comprises genotypes 2 and 17 which are low yielders (below the grand mean) with similar high interactions. The second sub cluster $(20,7$ and 19) is characterized by genotypes with low mean yield response less than the grand mean and with small positive and negative interactions. The splitting down of the second main branch resulted in three sub cluster levels. The first sub cluster of the second branch contained genotypes 1, 13, 3 and 14. These genotypes are high yielders with low interaction with the environments. The second sub branch included genotypes 12, 6, 10, 11, 15, 8, 9 and 5. Genotypes 18, 4 and 16 grouped in the third sub cluster of branch two. These two sub clusters are characterized by intermediate to high yield with high genotype by environment interactions adapted to specific environments.

When environmental clustering is considered, it has two main branches. The first cluster comprises of 7,10 and 8 and the remaining was included in the same second branch. The first branch is characterized by lower average yielders with high level of interaction. However, the second branch contains three intermediate to high yielding sub clusters. The first sub cluster included 1, 2, and 3 which are the same locations of different season characterized by intermediate yield potential and low interaction with the genotypes. Environments 4, 5 and 6 were included in the second sub cluster which is high yielding with high interaction with the genotypes. These locations are similarly characterized by high rainfall; that is, the reason they were clustered in the same branch. Environment 10 which is in the same location as environment 11 and 12 was classified as intermediate yielding environment with high interaction, environment 9 which is in the same location as environment 7 and 8 , classified in low yielding environment with low interaction was thus, classified differently. Environments 12, 9 and 


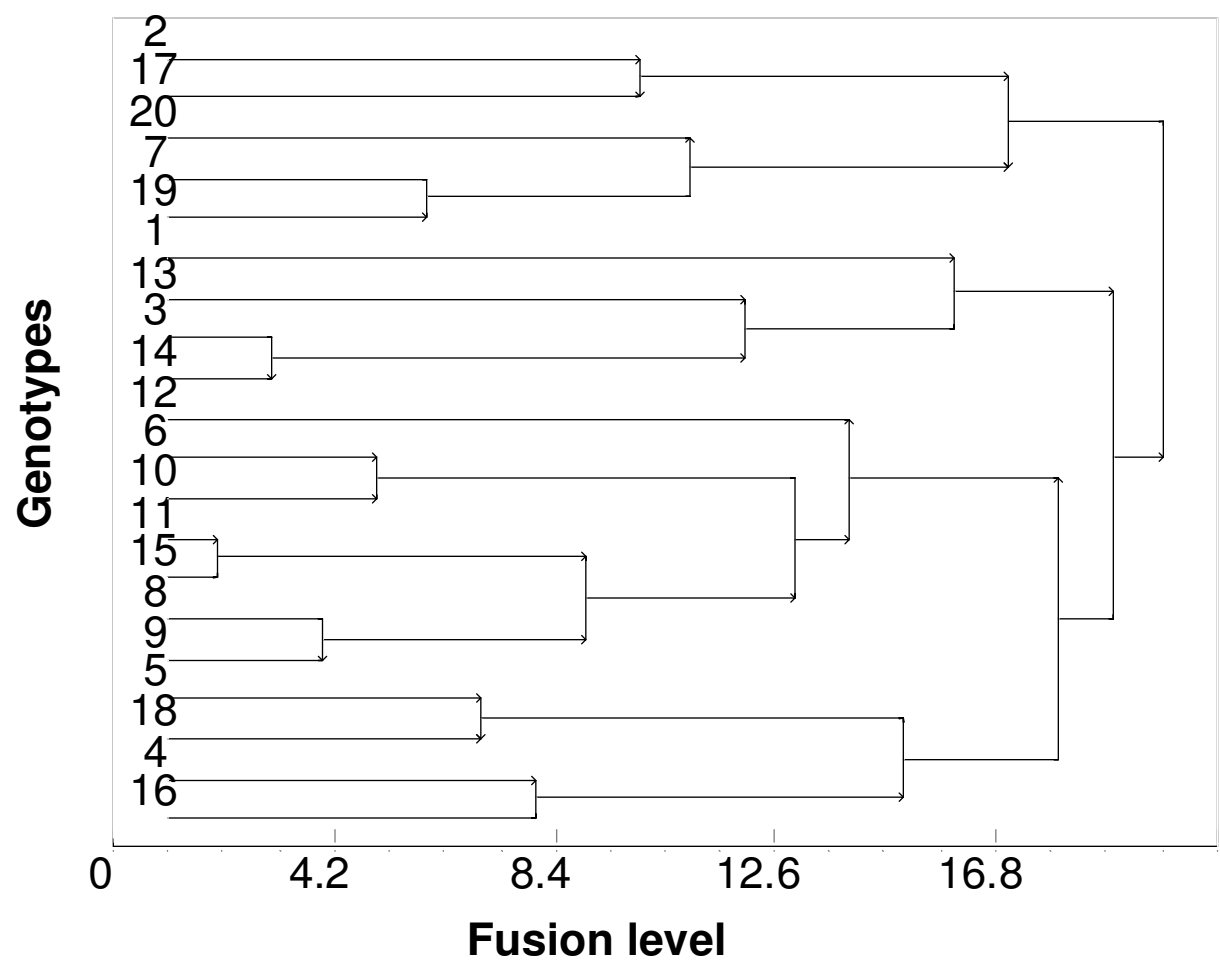

Figure 3. Dendrogram resulting from clustering of AMMI 2 adjusted genotype mean. $1=$ EH1609-F5.B3-10 (G1); 2 = 37622 (G2); 3 = 118173 (G3); 4 = Bekoji sel-8 (G4); 5 = 118007 (G5); 6 = EH1510-F6.10H.3 (G6); $7=108932$ (G7); $8=118173$ (G8); $9=118146$ (G9); $10=\mathrm{EH} 1746-\mathrm{F} 6 . \mathrm{B} 2-109$ (G10); $11=\mathrm{EH} 1606-\mathrm{F} 5 . \mathrm{B} 2-7$ (G11); $12=\mathrm{EH} 1601-\mathrm{F} 5 \cdot \mathrm{B} 2-2$ (G12); $13=$ EH1603-F5.B1-4 (G13); $14=$ EH1612-F5.B3.13 (G14); 15 = HB1533-sels (G15); $16=$ Miscal-2 (G16); $17=$ Miscal-1 (G17); $18=$ HB-242-sels (G18), $19=$ HB-52 (G19); 20 = HB-120 (G20).

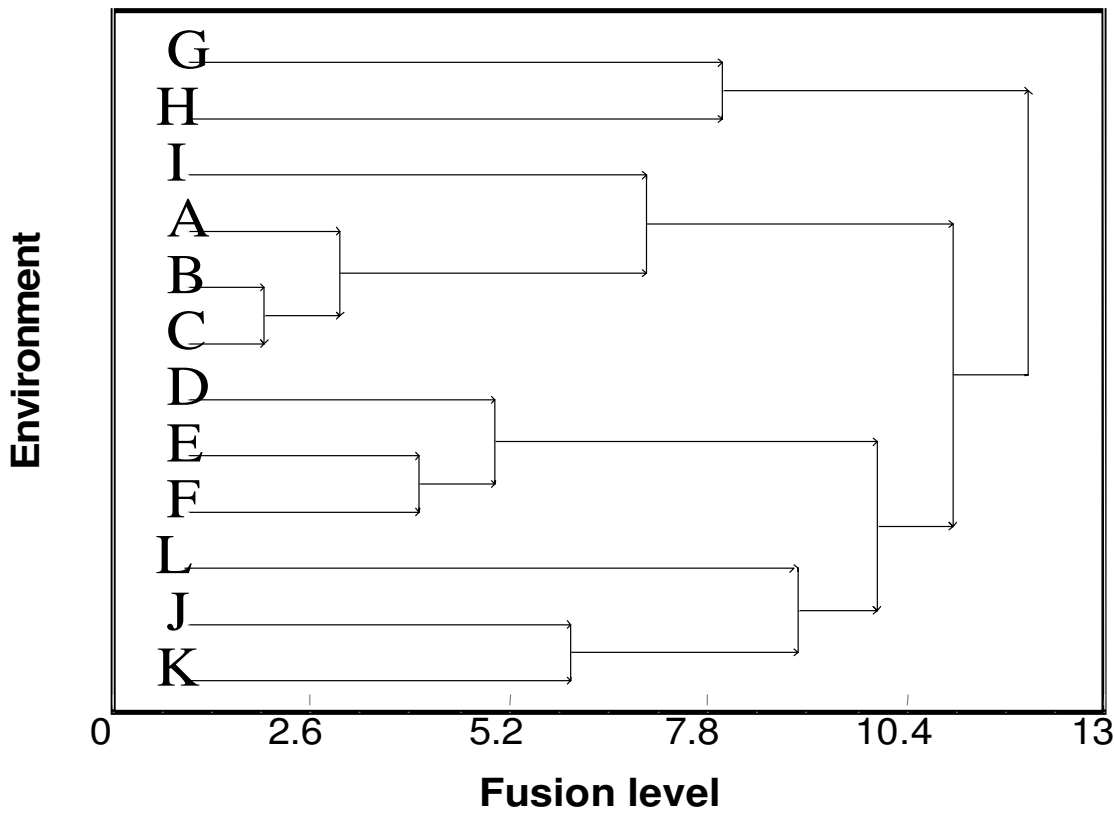

Figure 4. Dendrogram resulting from clustering of $A M M I 2$ adjusted environment mean. $\mathrm{A}=$ Adet, 2004; $\mathrm{B}=$ Adet, 2005; $\mathrm{C}=$ Adet, 2007; $\mathrm{D}=$ Debretabor, 2004; $\mathrm{E}=$ Debretabor, 2005; F = Debretabor, 2007; G = Dabat, 2004; H = Dabat, 2005; I = Dabat, 2007; J = Laygaint, 2004; K = Laygaint, 2005; L = Laygaint, 2007. 
Table 4. Rainfall, soil type and altitude of the locations.

\begin{tabular}{lccl}
\hline Location & Altitude (m.a.s.l) & Total annual rainfall $\mathbf{( m m})$ & Soil type \\
\hline Adet & 2240 & 1331.8 & Nitosol \\
Debretabor & 2630 & 1378.6 & Luvisol \\
Dabat & 2620 & 963.4 & Cambisol \\
Laygaint & 2500 & 950.4 & NA \\
\hline
\end{tabular}

Sources: NMSA, BBO; Tsige, 2002 and Yihenew 2004; NA, not available.

11 are third sub cluster of the second branch with low yield response to low level of interaction with genotypes. The first branch is characterized by low rainfall probably separated from the remaining environments. Sub cluster one and two were clustered in the same branch by cluster analysis. This could be attributed to similarities between the two locations (Adet and Debretabor) in rainfall and length of growing season (Table 4).

\section{DISCUSSION}

In crop improvement programs, genotypes are tested in different seasons and locations. These determined the performance and adaptation of genotypes. One obvious obstacle was the presence of noise and error in the field data resulting from GxE interactions and random errors. AMMI model is instrumental in identifying such components and making adjustments for yield estimates (Girma et al., 2000). Gauch (1988) explained the main features of multivariate models, which also include AMMI, and they account for a large proportion of pattern (variability) in their first few components, with subsequent dimensions accounting for a diminishing percentage of noise. Crossa et al. (1990) indicated that the noise in the AMMI analysis was quantified by the residual sum of square after adjusting for the best predictive model, whereas the error was estimated by the difference among individual experimental units (replicates) with the same treatment combination.

The AMMI model revealed that the two PCA axes account for the majority of the variation of GxE interaction. According to Girma et al. (2000), this could be associated with the nature of the crop, environmental characteristics or diverse genetic background obtained from different sources. Addition of the third, fourth or fifth axes may contribute to the accurate estimation of yield; however, based on AMMI analysis of variance, the first five IPCA AXES showed significant variation. The remaining axes have no real contribution in representing GxE interaction rather, most of it, is attributed to noise caused by different unpredicted factors.

This experiment also demonstrated the advantages of adding the AMMI model for the analysis of the GxE interaction for grain yield in malting barley. Simultaneous assessment of IPCA scores for genotypes and environments facilitates the interpretation and identifi- cation of specific interactions among them. For example, genotypes with a positive IPCA would be particularly adapted to environments with a positive IPCA and poorly adapted to environments with a negative IPCA (Gauch, 1992). G1 with a positive IPCA showed high adaptation to $A, B, C, D, E$ and $F$ environments, where it is ranked as a dominant genotype (Table 1). G13 with a negative IPCA was highly adapted to environments $\mathrm{G}, \mathrm{H}, \mathrm{I}, \mathrm{J}, \mathrm{K}$, and $L$ with negative IPCA.

AMMI 2 estimated values were used in the cluster analysis to test the diversity of genotypes and environments. The AMMI 2 estimated value for clustering and exhibited grouping of genotypes and environments into cluster after the interaction component was accounted for and thus it is a more precise clustering method.

\section{REFERENCES}

Crossa J, Fox PN, Pfeifer WH, Rajaram S, Gauch HG (1990). AMMI adjustment for statistical analysis of international wheat yield trial. Theor. Appl. Genet. 81:27-37.

Gabriel KR (1971). The biplot-graphical display of matrices with applications to principal components analysis. Biometrika 58:453467.

Gauch HG (1992). Statistical Analysis of Regional Yield Trials: Ammi Analysis of Factorial Designs. Elsevier, Amsterdam, The Netherlands, P. 278.

Gauch HG (1988). Model selection and validation for yield traits with interaction. Biometrics 44:705-715.

Gauch HG, Zobel RW (1996). AMMI analysis of yield trials. In: karg, M.S. and Gauch, H.G (eds). Genotype by environment interaction. CRC Press, Bocarator, Florida. pp. 85-122.

Girma T, Getachewu T, Bejiga G (2000). AMMI adjustment for yield

estimate and classification of genotypes and environments in field pea (Pisum sativus L.). J. Genet. Breed. 54:183-191.

Gollob HF (1968). A statistical model which combines features of factor analytics and analysis of variance techniques. Psychometrika 33:73115.

Kang MS (1990). Understanding and utilization of genotype by environment interaction in plant breeding. In: Kang, M.S. (ed.) Genotype by environment interaction and plant breeding. Louisiana state University agriculture center, Baton Rouge, Louisiana, USA. pp. 52-68.

Kempton RA (1984). The use of biplots in interpreting variety by environment interactions. J. Agric. Sci. 103:123-135.

Purchase JL (1997). Parametric analysis to describe GxE interaction and stability in winter wheat. PhD Thesis, Department of Agronomy, Faculty of Agriculture, University of the Orange Free State, Bloemfontein. South Africa.

Tsige G (2002). Genotype by environment interactions and stability analyses of Ethiopian Mustard. A Dessertation submitted in partial fulfillment of the requirements for the Degree of Doctor of Phylosophy, Graduate School, Freestate University. P. 200.

Yan W (1999). A study on the methodology of yield trial data analysis- 
with special reference to winter wheat in Ontario. PhD Thesis, University of Guelph, Ontario. Canada.

Yan W, Hunt LA, Sheng Q, Szlavnics Z (2000). Cultivar evaluation and mega environment investigation based on the GGE biplot. Crop Sci. 40:597-605.

Yan W, Rajcan I (2002). Biplot analysis of test sites and trait relations of soybean in Ontario. Crop Sci. 42:11-20.

Yihenew GS (2004). Modelling of nitrogen and phosphorus fertilizer recommendations for Maize (Zea mays L.) grown on alfisols of

northwestern Ethiopia. A Dessertation submitted in partial fulfillment of the requirements for the Degree of Doctor of Phylosophy /Tropical Agriculture/ Graduate School. Kasetsalt University. P. 220.
Zobel RW (1990). A powerful statistical model for understanding genotype by environment interaction. In M.S. Kang (ed.) Proc. Genotype by environment interaction and plant breeding. Louisiana State Univ. Baton Rouge. pp. 126-140.

Zobel RW, Wright MJ, Guach HG (1988). Statistical analysis of a yield trial. Agron. J. 80:388-393. 